\title{
ARTICLE
}

\section{Radiographic test problem for MCNP and other mesh-based applications}

\author{
Erik F. Shores ${ }^{*}$, Clell J. Solomon, John D. Zumbro, Duane P. Flamig and Guillermo Terrones \\ Los Alamos National Laboratory, Los Alamos, NM, 87545, USA
}

\begin{abstract}
Synthetic radiographs may be created with the Los Alamos Monte Carlo code MCNP6. A simple MCNP test problem, essentially a $10-\mathrm{cm}$ high truncated aluminum cone containing three $1-\mathrm{cm}$ radius tungsten spheres, each in turn containing a cylindrical void region, was devised to illustrate effects of various code options. While the radiographic test object (RTO) was initially constructed in MCNP's traditional combinatorial solid geometry (CSG), two additional mesh-based versions were created to exercise two new "hybrid-geometry" features of MCNP6. This paper describes the RTO, user input to exercise the new features and results from several versions of the RTO geometry. To further illustrate possibilities afforded by these new features, this paper also describes MCNP-simulated proton radiography of the CYCLOPS experiment as modeled by PAGOSA, a three-dimensional multimaterial hydrodynamics computer code developed at Los Alamos. CYCLOPS, essentially a line-wave generated, steel-confined, plastic-bonded high-explosive experiment in cylindrical geometry, resulted in a collection of 20 proton radiographs. Such data is extremely valuable for PAGOSA and MCNP6 code validation efforts; the former in terms of burn fronts and reflected shocks and the latter in terms of X-ray and proton transport.
\end{abstract}

\section{Keywords: Monte Carlo; MCNP6; unstructured mesh; radiography; proton}

\section{Introduction}

The "Flux Image Radiograph", or FIRn:p tally in MCNP [1] parlance, is shown schematically in Figure 1 and makes synthetic radiography applications possible with MCNP. Coupled with the general source definition and other tally features, the user defines an image grid with FSn and $C n$ entries and has great flexibility to model source-object-detector systems. Photon radiography is considered here but the FIR tally also works with neutrons.

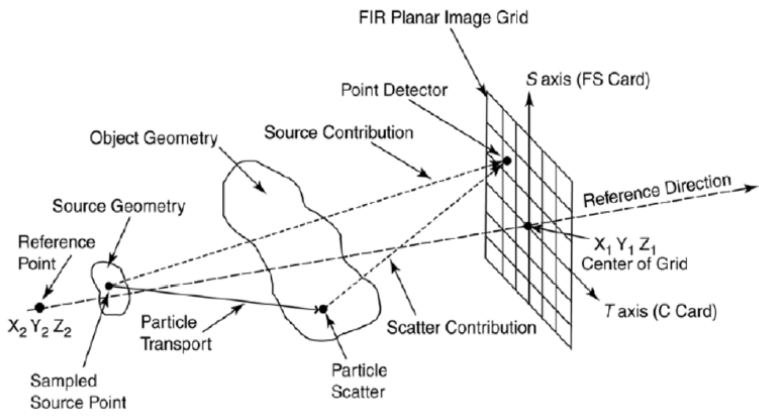

Figure 1. Flux image radiograph (FIR) tally illustration.

As geometries become more complicated (e.g. through use of coordinate transformations and/or rotations), the user may find unexpected tally results if

*Corresponding author. Email: eshores@lanl.gov the geometry and image grid coordinate systems are misunderstood. Directions of the image-plane-defining $s$ - and $t$-axes in Figure 1, for example, depend on the user-defined reference direction vector. Furthermore, post-processing tools (for visualizing tally output) may render images in an unexpected way. A simple test problem with clear orientation features was devised to investigate radiographic image production from various code options. Similar issues and test problems have been previously described [2-4] but inputs were not provided.

\section{Sample problem}

\subsection{Combinatorial solid geometry (CSG) option}

The sample problem is a truncated aluminum (2.7 $\mathrm{g} / \mathrm{cc})$ cone containing three tungsten $(18 \mathrm{~g} / \mathrm{cc})$ spheres of $1 \mathrm{~cm}$ radius; within each sphere is a cylindrical void region. A steel $(7.8 \mathrm{~g} / \mathrm{cc})$ sphere of $1 \mathrm{~cm}$ radius centered at $(\mathrm{x}, \mathrm{y}, \mathrm{z})=(5,5,5)$ is included for additional orientation. The relevant MCNP input file for the radiographic test object (RTO) is provided as an Appendix. A cross-sectional geometry slice, the default MCNP plot where the $y$-direction points to the right, the z-direction is vertical and the positive $\mathrm{x}$-direction points toward the viewer is provided in Figure 2. The problem origin for this right handed coordinate system is centrally located at $(y, z)=(0,0)$. 


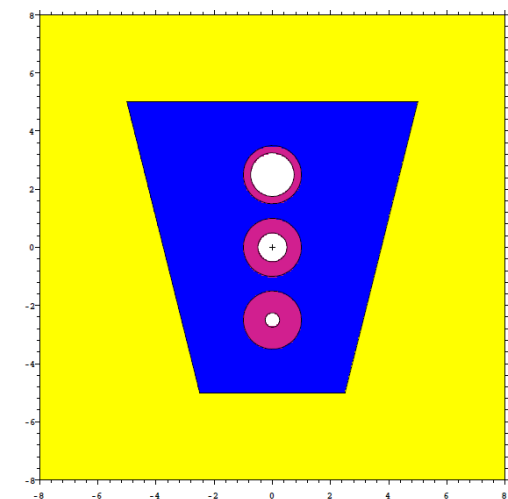

Figure 2. RTO geometry slice at $\mathrm{x}=0 \mathrm{~cm}$.

A three-dimensional view of the RTO, created with the Los Alamos graphics package OSOLOCO [5], is shown in Figure 3 with a portion removed to visualize the interior; the tungsten spheres, their cylindrical voids and the steel fiducial are readily identified.

The source position in the RTO specification is $(\mathrm{x}, \mathrm{y}, \mathrm{z})=(-133,0,0)$, directed through the test object in a direction orthogonal to the conical symmetry axis, and incident on a detector grid at $(\mathrm{x}, \mathrm{y}, \mathrm{z})=(392,0,0)$.

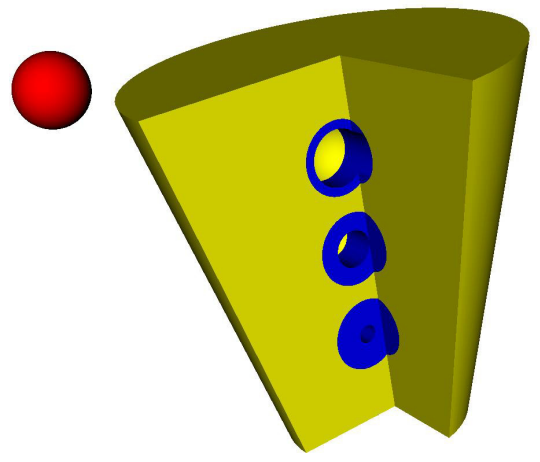

Figure 3. 3D perspective of the RTO from OSOLOCO.

\subsection{Hybrid geometry options}

The MCNP6 team recently added two powerful geometry features: 1) an unstructured mesh (UM) capability designed to exploit mesh geometries created with the ABAQUS/CAE tool, a suite of computer-aided engineering software applications and 2) the ability to create and/or "embed" structured mesh geometries in the form of LNK3DNT files. The latter, simply a formatted output specification, is intended to allow direct comparison of MCNP6 results (e.g., KCODE simulations) to those from the deterministic Los Alamos code PARTISN. A structured mesh version of the RTO was created along with an UM version from ABAQUS.

Besides an optional transformation entry, the CSG input file contains an optional input block to create, via MCNP6, a LNK3DNT version of this geometry. In this case, the binary file efslinktest was created with this command [6]:

monp6 m i=cone.i linkout=efslinktest

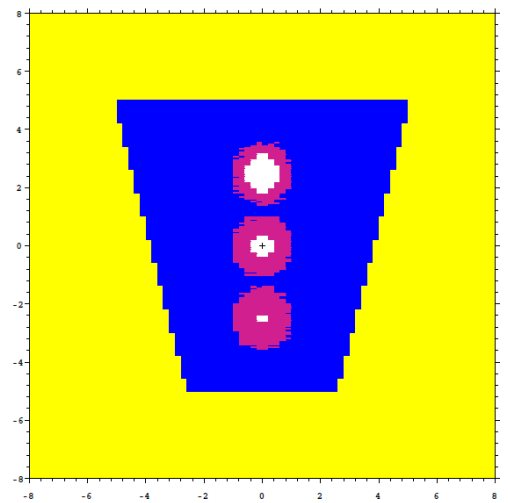

Figure 4. 2-mm mesh LNK3DNT version of the RTO.

and the resultant mesh is essentially a $16 \times 16 \times 16 \mathrm{~cm}$ cube of $80 \times 80 \times 80$ elements. The $2 \mathrm{~mm}(16 \mathrm{~cm} / 80$ mesh elements) "grid version" of this geometry is shown in Figure 4. A 1-mm LNK3DNT version of the RTO is a factor of 8 larger (98.3 MB vs 12.3 MB).

The UM option provides the ability to tally on mesh elements and the tetrahedral mesh RTO created with ABAQUS is shown in Figure 5 with tally results (e.g. energy deposition) to demonstrate visualization options.

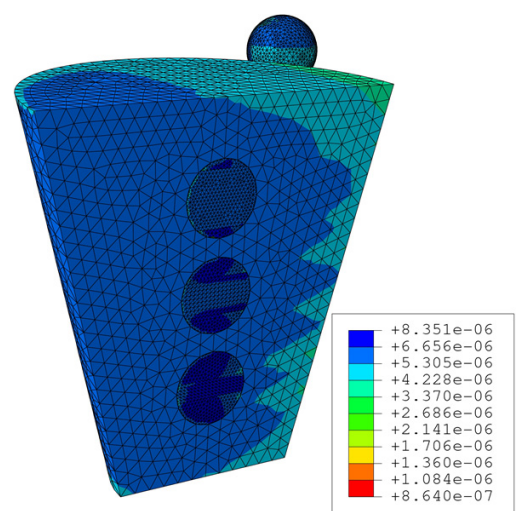

Figure 5. Tetrahedral mesh version of the RTO with tally.

\section{Radiographic results}

The nominal synthetic radiographic image ("direct" tally from a single photon history; i.e. ignoring scatter) from the CSG version of the RTO is shown in Figure 6. Radiographs are generally viewed from the source perspective and the steel sphere is thus expected in the upper left quadrant of the simulated image. Default image presentation was retained for Figure 6, however, and while it appears the viewer is looking toward the source, readers are reminded of the reference vector $(1,0,0)$ specified on the FIRn:p tally entry (see Figure 1).

Alternatively, the reference vector (and thus viewing perspective) could be modified by specifying the "coordinates defining outward normal to image grid" as (400 00 ) on the FIR entry, resulting in a reference vector of $(-1,0,0)$, but such an image requires $180^{\circ}$ rotation as demonstrated in Figure 7. In any case, such manipulations are readily handled in post-processing.

Interested readers are encouraged to explore scatter contributions in this RTO by extending photon physics 
and transport options. Image blurring from scatter, for example, becomes readily visible with as few as 100 histories. Additional options include making random offset (e.g. nonzero tenth entry on the FIR tally) contributions to each pixel or rotating the geometry through use of transformations as illustrated in Figure 8.

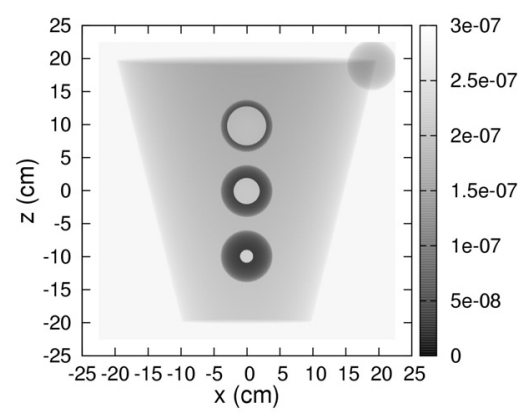

Figure 6. Nominal RTO synthetic radiograph requires horizontal pixels to be reversed; reference vector is $(1,0,0)$, $\mathrm{s}$-axis is $(0,1,0)$ and $\mathrm{t}$-axis is $(0,0,1)$.

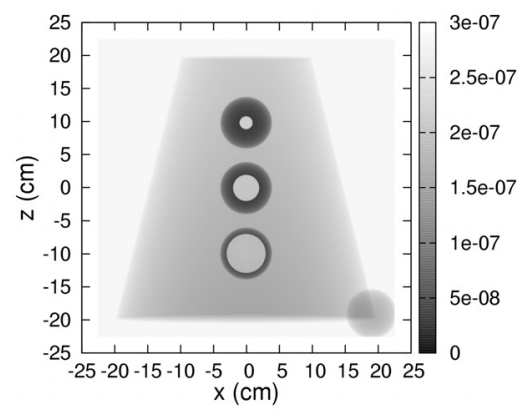

Figure 7. RTO synthetic radiograph with alternate reference vector specification requires image rotation; here reference vector is $(-1,0,0)$, s-axis is $(0,1,0)$ and $t$-axis is $(0,0,-1)$.

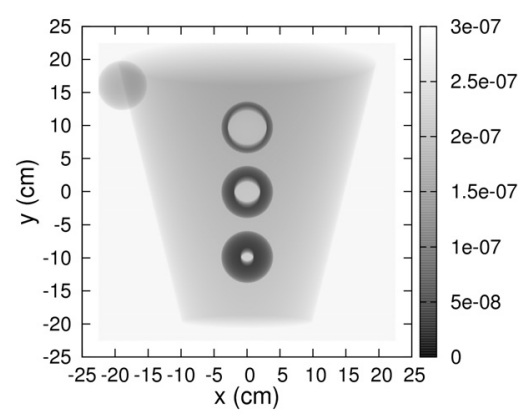

Figure 8. Small end of cone toward source 8 degrees.

Arbitrary intensity units are shown in Figures 6-8 but are the same for each figure. In short, for an MCNP user to understand the relationship between the geometry coordinate system and that of the detector grid, MCNP's output file should be studied-especially the output comments highlighting relevant vectors.

Comparisons were made between the hybrid and CSG radiographs and two examples are shown in Figure 9 (number of standard deviations different from the CSG) and Table 1. Despite having a factor of 20 more elements than the ABAQUS version (4,096,002 versus 180,816), the structured mesh LNK3DNT RTO did not represent the 10 cell CSG model as well. On a computer time per cell basis, however, the UM geometry was a factor of 16 greater than that for LNK3DNT. This is not surprising considering the more difficult tracking in the UM. All problems ran 5e9 histories with 128 MPI tasks and consistent rendezvous at $1 \mathrm{e} 9$ histories.

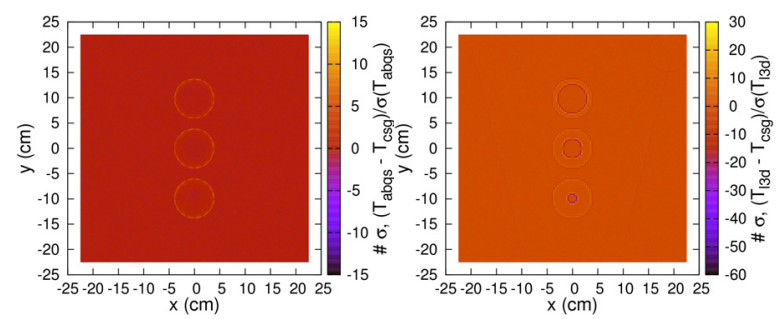

Figure 9. ABAQUS (1.) and LNK3DNT (r.) compared to CSG.

Table 1. RTO problem comparison.

\begin{tabular}{rccc}
\hline Problem & Wall Time $(\mathrm{min})$ & Avg. Tally & Rel. Err. \\
\hline CSG & 16.73 & $2.44794 \mathrm{e}-7$ & $1.94590 \mathrm{e}-5$ \\
ABAQUS & 268.52 & $2.45041 \mathrm{e}-7$ & $1.94475 \mathrm{e}-5$ \\
LNK3DNT & 364.92 & $2.45076 \mathrm{e}-7$ & $1.94599 \mathrm{e}-5$ \\
L3D2MCNP & 393.65 & $2.45996 \mathrm{e}-7$ & $1.94153 \mathrm{e}-5$ \\
\hline
\end{tabular}

\section{CYCLOPS: a practical mesh application}

In the Los Alamos CYCLOPS experiment, proton radiography was used to investigate evolution of the burn front and associated reflected shocks on a high explosive (HE) charge confined between an outer cylindrical steel liner and an inner elliptical tin liner. The charge was initiated such that a large region of the HE was not within the line of sight of the detonation line and thus provided a suitable experimental platform to test various burn models and EOS formulations. Furthermore, the off-axis initiation allowed burn fronts to travel around the charge through different confining paths. Simulations by one of the authors (GT) with the PAGOSA code were performed to assess the accuracy of several HE burn methodologies [8] and several mesh resolutions were considered: $1.0,0.7$ and $0.5 \mathrm{~mm}$. The latter is shown in Figure 10 in MCNP geometry along with a synthetic proton radiograph made by one of the authors (JDZ) using an FMESH tally [9]. A utility was
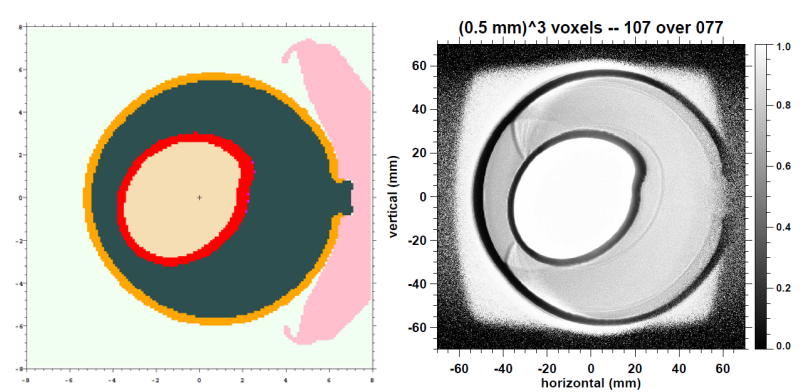

Figure 10. MCNP6 geometry (left) and synthetic proton radiograph of the CYCLOPS experiment. 
written by another author (CJS) to manipulate PAGOSA output into LNK3DNT format to embed the geometry; alternatively, CSG versions of the structured mesh can be simply made with an appropriate number of planes or, more efficiently, by utilizing MCNP's lattice construct.

\section{Conclusions}

MCNP users have great flexibility to model source-object-detector systems for radiographic applications. An RTO was devised to exercise MCNP's radiographic tally options and illustrate proper image orientation. MCNP's output description of the $s$ - and $t$ axes, coupled with the tally reference vector, are key to understanding FIRn:p tally output. This work corroborates Temple's conclusions [4] and the authors endorse his proposed modifications to the MCNP manual regarding his "better description for the radiography tally". Relative to traditional CSG, new "hybrid geometry" features in MCNP6 exploit structured and unstructured mesh versions of the problem and this note provides a practical example.

\section{Acknowledgements}

The authors would like to thank Monte Carlo Team members Larry Cox, Art Forster and Jeremy Sweezy (XCP-3) for useful discussions on this topic and Laura Lang (HPC-1) for OSO collaboration. Terry Adams and Steve Nolen (XCP-3) provided timely MCATK assistance and CJ Solomon's (XCP-7) MCTALTOOL L3D2MCNP utilities are greatly appreciated.

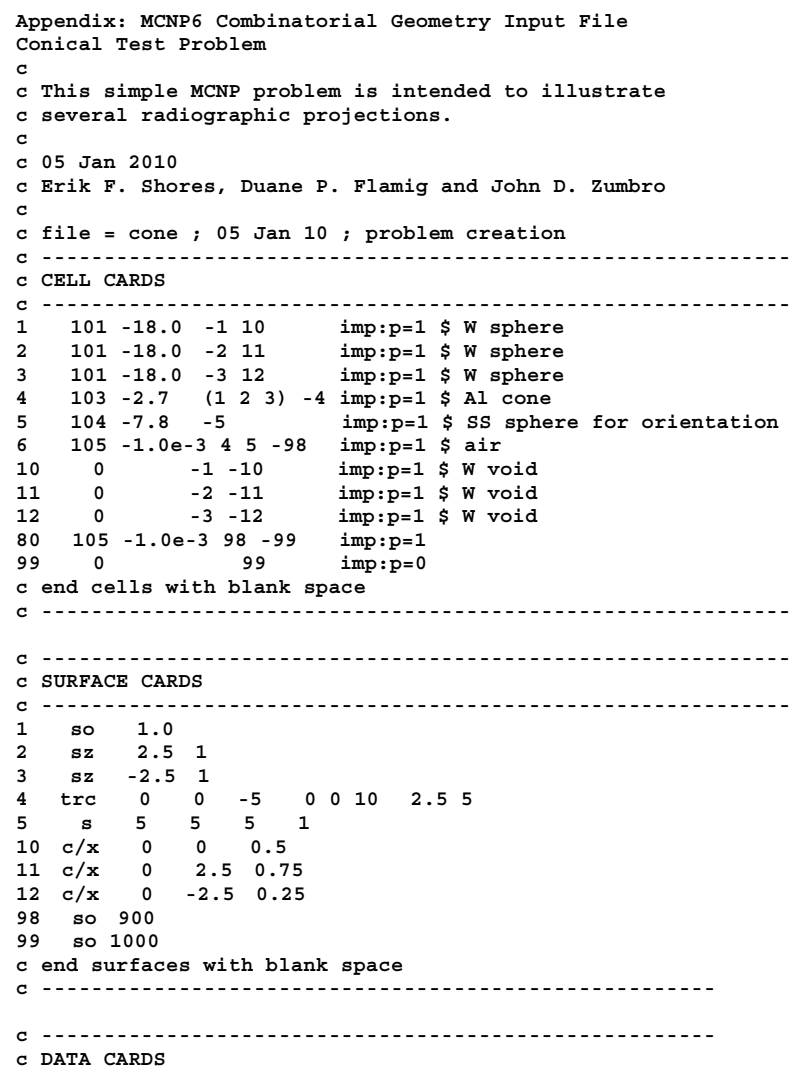

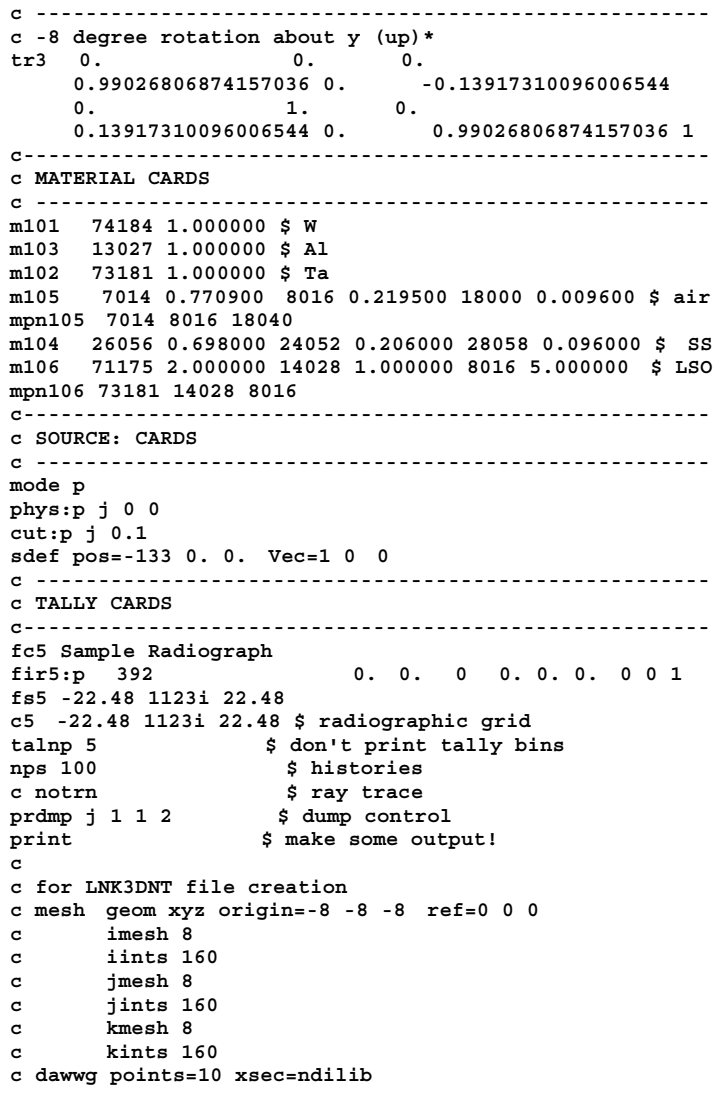

\section{References}

[1] X-5 Monte Carlo Team, MCNP - A General Monte Carlo N-Particle Transport Code, Version 5, Volume 1: Overview and Theory, internal Los Alamos report LA-UR-03-1987 (24 Apr. 2003, revised 01 Feb. 2008).

[2] B. Temple, MCNP5 Radiography Tally Input Modifications, internal Los Alamos report LA-UR-04-0910 (06 Feb. 2004).

[3] B. Temple, Verification of Consistency Between Radiography and FMESH Tallies for Simulated $X$-Ray Radiographs in MCNP, unpublished (22 Dec. 2004).

[4] B. Temple, Addendum to LA-UR-04-0910, A Clarification of the MCNP5 Radiography Tally Directions, internal Los Alamos report LA-UR-06-8345 (29 Nov. 2006).

[5] L. Lang, OSOLOCO 1.1.2, A Constructive Solid Geometry (CSG) Editor, LA-CC-11-070 (2011).

[6] L. Cox, LNK3DNT Geometry Support: User Guidance for Creating and Embedding, internal Los Alamos report LA-UR-11-01654 (2011).

[7] XCP-3 Monte Carlo Team, MCATK: Monte Carlo Application Toolkit, personal communications (2011).

[8] G. Terrones, M. W. Burkett and C. Morris, Burn front and reflected shock wave visualization in an inertially confined detonation of high explosive, Proc. of the International Conference of the APS Topical Group on Shock Compression of Condensed Matter, Chicago, IL, 26 Jun-01 Jul. 
2011, 1426 (2012), pp. 239-242.

[9] J.D. Zumbro, A. Acuff, J.S. Bull, H.G. Hughes, R.E.

Prael and E.C. Selcow, Proton radiography applications with MCNP5, Rad. Prot. Dosimetry, Vol 117, No 4, (2005), pp. 447-452. 